\title{
Assessment of Malaria Intermittent Preventive Treatment (Ipt) Uptake among Pregnant Women attending Antenatal Care at Kasanje Health Center III, Wakiso District
}

\author{
Doreen Kabatongole ${ }^{a, 1}$, Moses Ochana \\ ${ }^{a}$ Faculty of Health Science, Uganda Martyrs University
}

\begin{abstract}
\end{abstract}

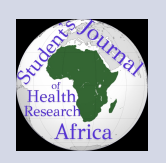

Background: ${ }^{a}$

Malaria in pregnancy is a major public health problem with substantial risks for both the mother and unborn child. Malaria is responsible for 10,000 maternal deaths globally, $20 \%$ of stillbirths, and $11 \%$ of newborn deaths in subSaharan Africa. The study intends to explore the extent of intermittent preventive treatment IPTp uptake among pregnant women attending ANC.

Methodology

A Cross-sectional survey was carried out among pregnant women attending antenatal care at Kasanje Health Center III to explore the extent of intermittent preventive treatment (IPTp) uptake among pregnant women attending ANC. Data on their socio-demographic characteristics and antenatal service utilization were collected using a questionnaire and review of antenatal care (ANC) cards. In-depth interviews were conducted and data on ANC service delivery collected and analyzed. Univariate, Bivariate, and multiple logistic regression analyses were done to determine factors associated with uptake of IPTp.

Results:

A total of 75 pregnant women were interviewed, all in reproductive age 15-48 years, with an average age of 25.6years. All the respondents (100\%) had attended antenatal care at least once. $58 \%$ of the participants wherein their third trimester. Many, $31 \%$ were on their second visit, $24 \%$ on their 3 rd visit while only $10 \%$ were on their first visit. The majority of the participants (88\%) had their ANC started in the first trimester. It was also noted that the number of IPTp varied significantly with the number of ANC visits (chi2=33.8, $p<0.001)$. The majority $(62.67)$ of the participants knew IPTp and the majority of the respondents (99\%) knew how malaria is transmitted. Abortion was mentioned as the major outcome of malaria in pregnancy(95\%) followed by Stillbirths(72\%).

\section{Conclusion and recommendations:}

An increased number of ANC visits, providing IPTp information to pregnant women, and the gestational age of pregnancy resulted in increased uptake of IPTp.

\footnotetext{
a email: kabsdor@gmail.com date submitted: 4th/04/2021 date accepted: 19 th/04/2021
}

\section{Background of the Study}

The study intends to explore the extent of intermittent preventive treatment IPTp uptake among preg- 
nant women attending ANC. World health organization defines intermittent prevention treatment of malaria in pregnancy (IPTp) as a full therapeutic course of antimalarial medicine given to pregnant women at routine antenatal care visits, regardless of whether the recipient has malaria or not as recommended by WHO and Uganda national malaria control program/policy.

Malaria is a parasitic infection transmitted by a bite of an infected female anopheles mosquito (Lagerberg, 2008). Malaria in pregnancy is a major public health problem with substantial risks for both the mother and unborn child. Malaria is responsible for 10,000 maternal deaths globally, $20 \%$ of stillbirths, and $11 \%$ of new-born deaths in sub-Saharan Africa(WHO, 2018). However, coverage of malaria prevention, screening, and treatment among pregnant women remains low in many countries in Sub-Saharan Africa(Agarwal et al., 2015). Uganda ranks 8th among the most affected countries in sub-Saharan Africa and has one of the highest malaria transmission rates in the world (USAID, 2019).

In 2017-2018, there were 219 million cases of malaria with an estimated 435,000deaths (WHO, malaria report 2018). The African region carries the highest malaria burden(WHO, 2018). Pregnant women and children under five years are among the most vulnerable group with the highest morbidity and mortality(Guinovart et al., 2006). Pregnant women infected with malaria usually have more severe symptoms and outcomes. The most common effects of malaria in pregnancy are miscarriage, intrauterine demise, premature delivery, low-birth-weight neonates, neonatal death, severe anemia, and maternal death(Ekejindu et al., 2006). Malaria can be prevented with the appropriate use of drugs, bed nets treated with insecticide, and effective educational outreach programs(Schantz et al., 2009).

The global distribution of gross domestic product shows a striking correlation between malaria and poverty. This includes effects on fertility, population growth, saving and investment, worker productivity, absenteeism, premature mortality, and medical costs(Ndyomugyenyi et al., 1998). Some of the socio and economic factors associated with malaria include low education and knowledge, socio-cultural barriers, malnutrition, concurrent infections (Ricci, 2012). Unless additional efforts are directed towards addressing access barriers among the pregnant women and the vulnerable, malaria will remain a major cause of morbidity and mortality in malaria-affected countries like Uganda.

Uganda's Malaria Control Strategic Plan identifies IPTp as one of the key strategies to prevent malaria in pregnancy. According to York et al., (2015), It is delivered as part of the focused ANC package and has been implemented countrywide since 2002. Yet, despite having made significant progress, Uganda did not meet the government's target of 85 percent of pregnant women receiving two doses of IPTp by the end of 2015. In 2014-15, less than half of pregnant women in Uganda received two or more doses of IPTp, despite overall one time ANC attendance being 94 percent in Uganda

Intermittent preventive treatment (IPTp) is a therapeutic course given to reduce maternal malaria episodes, maternal and fetal anemia, placental parasitemia, low birth weight, and neonatal mortality. This is critical considering that malaria infection during pregnancy is associated with significant risks for both the mother and her unborn child. In malaria-endemic countries like Uganda; in addition to IPTp WHO recommends a complete package that includes, promotion and use of insecticidetreated nets (ITNs) and appropriate case management through prompt and effective treatment of malaria in pregnant women. (Mubyazi et al., 2008).

The current WHO recommendations for Intermittent Prevention Treatment (IPTp) of malaria include starting the therapy as early as possible in the second trimester. The pregnant women should take IPTpat for each scheduled antenatal care (ANC) visit until the time of delivery provided that the doses are given at least one month apart. The therapy should ideally be administered as directly observed therapy (DOT) of three tablets Sulfadoxine/Pyrimethamine (SP) (each tablet containing 500 $\mathrm{mg} / 25 \mathrm{mg} \mathrm{SP}$ ) at each antenatal visit. Sulfadoxine/Pyrimethamine should not be given during the first trimester of pregnancy; however, the last dose of IPTP-SP can be administered up to the time of delivery without safety concerns. Furthermore, SP is contraindicated in women receiving co-trimoxazole prophylaxis due to a higher risk of drug interaction(WHO, 2013). After widespread malaria parasite resistance to antimalarial agents, artemisinin-based combination therapy has also been introduced as IPTp for malaria in pregnancy and it's given during the second and third trimester. Dihydroartemisinin-piperaquine (DHA- 
$\mathrm{PQ}$ ) is a promising new fixed oral combination given as a three-day course in IPTp(Poespoprodjo et al., 2014).

The barriers to IPTp-Sp uptake include Inaccurate estimation of gestational age that leads to missed doses, unclear communication on the timing of doses, and the recommended number of SP doses required during pregnancy (Mubyazi et al., 2008). Furthermore, late or no antenatal care attendance and poor implementation of directly observed administration of SP (Ouma et al., 2007). In Uganda, African Strategies for Health (2016) noted misconceptions of not giving SP on an empty stomach, poor counseling on the use of SP, and clients' familiarity with mosquito nets rather than IPT alone as some of the facility-level factors influencing the rate of uptake of IPTp-SP. Pregnant women and society associate, SP with severe adverse outcomes such as abortion, skin reaction, and lack of antifever effect. SP can cause effects such as StevensJohnson syndrome in people who are allergic to sulfur with possible dramatic and potentially fatal effects. However, such side-effects are rare and in most cases exaggerated. The perception people have of a drug may influence their acceptance of the medicine. A drug that is bitter or associated with abortion in some cultures is likely to be less complied with by the target population. The situation is made worse when health service providers share such sentiments. In Tanzania, some health professionals expressed their doubts about the rationale for recommending SP for malaria treatment in areas where drug resistance is already high. In Malawi, some health workers believe that SP is not appropriate to be taken by a person with an empty stomach(Mubyazi et al., 2008).

The success of any malaria intervention depends on consumer and provider compliance, safety, cost, health care delivery system, and degree of integration with other interventions. Institutionalization of public health interventions within constrained infrastructure, other supply and demand conditions is critical to the effectiveness of such interventions (Mubyanzi et al., 2008).

This raises concerns about health policy programs targeting services to populations in need. In western Kenya, Improved uptake of IPTp-SP was associated with a rural setting, with a lower level of education, and distance to the facility where ANC is provided (Sangaré et al., 2010). The challenges facing IPTp uptake are however not clearly described in Uganda. This study, therefore, intends to establish the rate and factors affecting IPTp uptake among pregnant women attending ANC at Kasanje Health Center iii, Wakiso district.

The Ugandan government adopted IPTp from World Health Organization (WHO) to help reduce the risk of malaria in pregnant women. This intervention involves the administration of three doses of SP as DOTS to pregnant women during the second and third trimester. This has been done to augment other key traditional interventions such as the use of insecticide-treated mosquito nets, indoor residual spraying, early diagnosis, and treatment of malaria. However, despite the high use of ANC services in many districts of Uganda, the extent of IPTp uptake remains unknown in most hospitals in Uganda, especially in a rural setting. This has exposed pregnant women to malaria and the associated unwanted outcomes such as miscarriage and intrauterine growth restriction, as well as pre-term and low birth weight delivery. Interventions have been put in place like conducting health education talks about the importance of IPTp use in pregnancy, VTHs have sensitized all pregnant women in the communities about IPTp uptake and availability of free IPTp services has been offered but this has yielded no efforts as the hospital has continued recording low IPTp coverage. Therefore, this study aims to assess the extent of IPTp uptake and associated factors among pregnant women attending antenatal care at Kasanje Health Center III, Wakiso district as there is no readily available information

The purpose of this study was to explore the extent of IPTp uptake and associated factors among pregnant women attending antenatal care at Kasanje Health Center III, so that uptake of IPTp among pregnant women is improved.

\section{Research Design \\ 2 Methodology}

The study design was cross-sectional and descriptive, employing a quantitative data collection method. It was a cross-sectional type of design because it involved the collection of data from a single point in time and from a group of respondents whose characteristics such as age, level of education, occupation, and marital status were described. The design was descriptive because it described the data without any changes in it. 


\section{Study setting}

The study was carried out in Kasanje health center III, Wakiso District which is found in Central Uganda. Kasanje Health center III is located in the southern part of the Wakiso district. It is located approximately 45.5 kilometers by road, west of Mpigi and approximately 18 kilometers northwest of Kampala. Kasanje Sub County had an estimated population of 20000 people who are predominant business people. Kasanje health center III provides general health care to all patients, antenatal care, deliveries, postnatal care, family planning, immunization, HIV screening, and care. The study setting was selected because the problem under study had been noted by the researcher and the researcher is a resident of Kasanje Sub County.

\section{Study population}

The target population was 73 consenting pregnant women who were attending the antenatal clinic at Kasanje Health Center III.

\section{Sample size and selection}

The sample size was estimated using the Cochran formula, $n=(Z 2 p q) / d 2$, where $n$ is the sample size, $Z$ is the $Z$-score that corresponds with the $95 \%$ confidence interval (1.96), $P$ is the proportion of antenatal attendants who received IPT 4 in 2020 $(5 \%,=0.05)$, $q$ is the proportion of antenatal attendants who received less than three doses of IPT $(1-0.05,=0.95)$, and $d$ is margin of error set at $5 \%$ (0.05)(Cochran, 2007). The estimated sample size, $\mathrm{n}$, is 73 .

\section{Sampling procedure}

The participants for the study were selected using the purposive sampling method. The sample size was 73 pregnant women, the researcher selected 10 participants from the available pregnant women who came for antenatal services each day in the health center during data collection and those who were eligible (that is; above 18 years) and willing to participate in the study were then enrolled. This was done for eight days.

\section{Inclusion criteria}

Subjects to be recruited into the study had to fulfill the following criteria; had at least one antenatal clinic visit before the interview and were only pregnant women.

\section{Criteria for exclusion}

Registering for the first time in the health facility and disabilities that disallow responses to questionnaire.

\footnotetext{
Definition of variables
}

The independent variables were factors associated with IPTp uptake among pregnant women attending ANC at Kasanje health center III.

The dependent variable was the prevalence of malaria uptake among pregnant women attending ANC at Kasanje health center III.

\section{Data Collection Methods}

One-on-one interviews were held with the pregnant women and data on background characteristics including age, education, number of children, occupation, and marital status were collected from them onto a questionnaire designed specifically for this study. The ANC cards of the mothers were also be reviewed and information on gestational age at the first ANC visit, number of visits, number of doses of IPT doses taken before delivery, and the gestational age at which the first and subsequent doses of IPT that were taken were extracted. Other data extracted from the ANC card included malaria infection during the most recent pregnancy, questions on why they did not receive the required IPT and why it was missed were also asked and recorded. Data on the prevalence of malaria was obtained from the mother's antenatal book and all results of malaria tests were extracted from the antenatal card and recorded. Where there was a discrepancy between the information given by the mother and what is documented, the information on the ANC card was used. The interviews were conducted in the local languages mainly Luganda and English by the researcher. The questionnaire was in English and so the information was recorded in English.

\section{Data collection procedure}

The researcher was accompanied and introduced to the respondents by the in-charge of Kasanje Health center III before approaching and collecting data from respondents. She first introduced herself to the respondents and explained the purpose and objectives of the study. The researcher administered questionnaires to respondents in the health unit. This improved efficiency and confidentiality during data collection.

\section{Data Collection Instrument}

Data was collected by the use of a questionnaire. This tool was used because it allowed for fast, efficient, and confidential data collection.

\section{Data processing}

The questionnaire responses were edited for accuracy without changing the meaning given in the 
response. Responses were manually coded and arranged properly for presentation and analysis.

\section{Data Management and Analysis}

The data was coded and entered into a computer database using EPI INFO 2002 statistical software(Kuczmarski, 2002). Data was then stored in excel 2007 and later imported to Stata SE for analysis. Percentages or means and standard deviations were computed for baseline characteristics of women interviewed. Cross tabulations to identify important relationships between variables were done. The relationship between sociodemographic characteristics of the women and their use or non-use of intermittent preventive treatment of malaria during pregnancy will be examined through bivariate and multivariate analysis, by computing odds ratio at $95 \%$ confidence level. A p-value $<0.05$ was considered as statistical significance.

\section{Ethical consideration}

The researcher got a letter of introduction from the Dean's office as a pre-requisite for carrying out the study. Then the researcher got permission from the District Health Officer of Wakiso district to enable data collection at the health center. The district health officer introduced the researcher to the in-charge of Kasanje health center III who then introduced the researcher to the participants. Consent of the participants was obtained after the purpose and objectives of the study had been identified and well explained to the participants. The study was purely for academic purposes and all the information given was treated with confidentiality and numbers instead of names were used to identify the respondents

\section{Dissemination of Results}

The results were compiled and recommendations are drawn in the report which was forwarded to Uganda Martyrs University for marking as it is one of the requirements for the award of a degree in Midwifery science, a copy was given to the district health officer of Wakiso district for reference and to also help them on how they can go about the challenges, a copy to Kasanje Health center III and the researcher also retained a copy for future reference and ownership.

\section{limitations of the study}

The Sample Size: Due to limitations in resources especially funds and time, as well as using a small sample size the results could not be generalized for other similar areas.
The quality of data: Due to reliance on mothers to provide all the required data for the study, this created limitations as some participants feared to open up and give the required data for the study, thus affecting the quality of the study. This was solved by a questionnaire with simple and clear questions.

Resources of both money and time during covid19 pandemic where everything was hiked including transport fares, accommodation, and others amidst financial constraints.

Despite all the expected limitations and challenges, the results of the study provided a meaningful basis on the assessment of malaria intermittent preventive treatment uptake among pregnant women attending antenatal care at Kasanje health center III.

\section{Results}

\section{Description of the Sample}

A total of 73 pregnant women were interviewed. All women were in the reproductive age group between 15 and 48 years. The mean (+/-SD) age of the respondents was $25.6(+/-6.5)$ years, with a median age of 24 years.

Most of the respondents, (30.7\%) had attained a secondary level of education, $26 \%$ primary level and a few made it at the university. Catholics (33\%), Anglicans (26.7\%), and Muslims (22.7\%) were the main religious dominations. The majority (65.3\%) of the participants were currently not married/once married. Most of the respondents (65\%) were students or unemployed. The majority of the respondents had attained secondary education and more (table 1)

\section{TABLE 1: Distribution of Age and Education Level of Study Participants}

All women were in the reproductive age group between 15 and 48 years. The mean (+/-SD) age of the respondents was $25.6(+/-6.5)$ years, with a median age of 24 years. Most of the respondents, (30.7\%) had attained a secondary level of education, $26 \%$ primary level and a few The majority of the respondents(60\%) had attained secondary education and more. 
Table 1. Distribution of Age and Education Level of Study Participants

\begin{tabular}{|c|c|c|c|}
\hline & & Frequency $(n=73)$ & Percent \\
\hline \multirow{4}{*}{ AGE-GROUPS } & $15-19$ & 16 & 21.3 \\
\hline & $20-24$ & 22 & 29.3 \\
\hline & $25-29$ & 19 & 25.3 \\
\hline & Above 29 & 18 & 24 \\
\hline \multirow{4}{*}{ EDUCATION LEVEL } & None & 10 & 13.3 \\
\hline & Primary & 20 & 26.7 \\
\hline & Secondary & 23 & 30.7 \\
\hline & $\begin{array}{l}\text { post sec- } \\
\text { ondary }\end{array}$ & 22 & 29.3 \\
\hline
\end{tabular}

\section{TABLE 2: distribution of religious affiliations of the study participants}

Catholics (33\%), Anglicans (26.7\%) and Muslims $(22.7 \%)$ were the main religious dominations.

\section{TABLE 3: Distribution of} Marital Status and Parity of the Study Participants

Majority (65.3\%) of the participants were currently not married/once married. $40 \%$ had one child, $23 \%$ had 2 children and $34.6 \%$ had 3 and more children

\section{TABLE 4: Distribution of Religious Affiliations of the Study Participants}

The majority of the participants (56\%) were unemployed/students, only $25 \%$ were self-employed and the rest (19\%) were either government or nongovernment employees.

Research Question One: What were the Factors Associated with IPTp Uptake among Pregnant Women Attending ANC at Kasanje Center III?

Pregnancy, Malaria and Intermittent Preventative Treatment

The mean gestational (+/-SD) age of the respondents was $26.8(+/-7.0)$ weeks with a minimum gestational age of 8 and a maximum of 38 weeks. The majority of the respondents were multigravidas (69\%) and the rest 30\% were primgravidas. The mean number of children produced by the respon- dents (+/-SD) was $2(+/-2)$ although the majority (40.4\%) had 1 child.

All the respondents (100\%) had attended antenatal care at least once during the most recent pregnancy and the majority (79\%) were attended to by the midwives. $58 \%$ of the participants wherein their third trimester. Many of the 31\% were on their second visit, $24 \%$ on their 3rd visit while only $10 \%$ were on their first visit. The majority of the participants (88\%) had their ANC started in the first trimester. It was also noted that the number of IPTp varied significantly with the number of ANC visits (chi2=33.8, $p<0.001$ )

The majority (62.67) of the participants knew IPTp and the majority of the respondents (99\%) knew how malaria is transmitted (Figure 1). Abortion was mentioned as the major outcome of malaria in pregnancy $(95 \%)$ followed by Stillbirths(72\%). (figure 2).

\section{Figure 1: Reported Mode of Transmission of Malaria.}

Most of the participants had taken 1 or 2 doses of fasindar (figure 6), however most (83\%) of them reported the drugs were not always available at the health facility (figure 7).

\section{Figure 3: Uptake of IPTp by Participants during the Current Pregnancy}




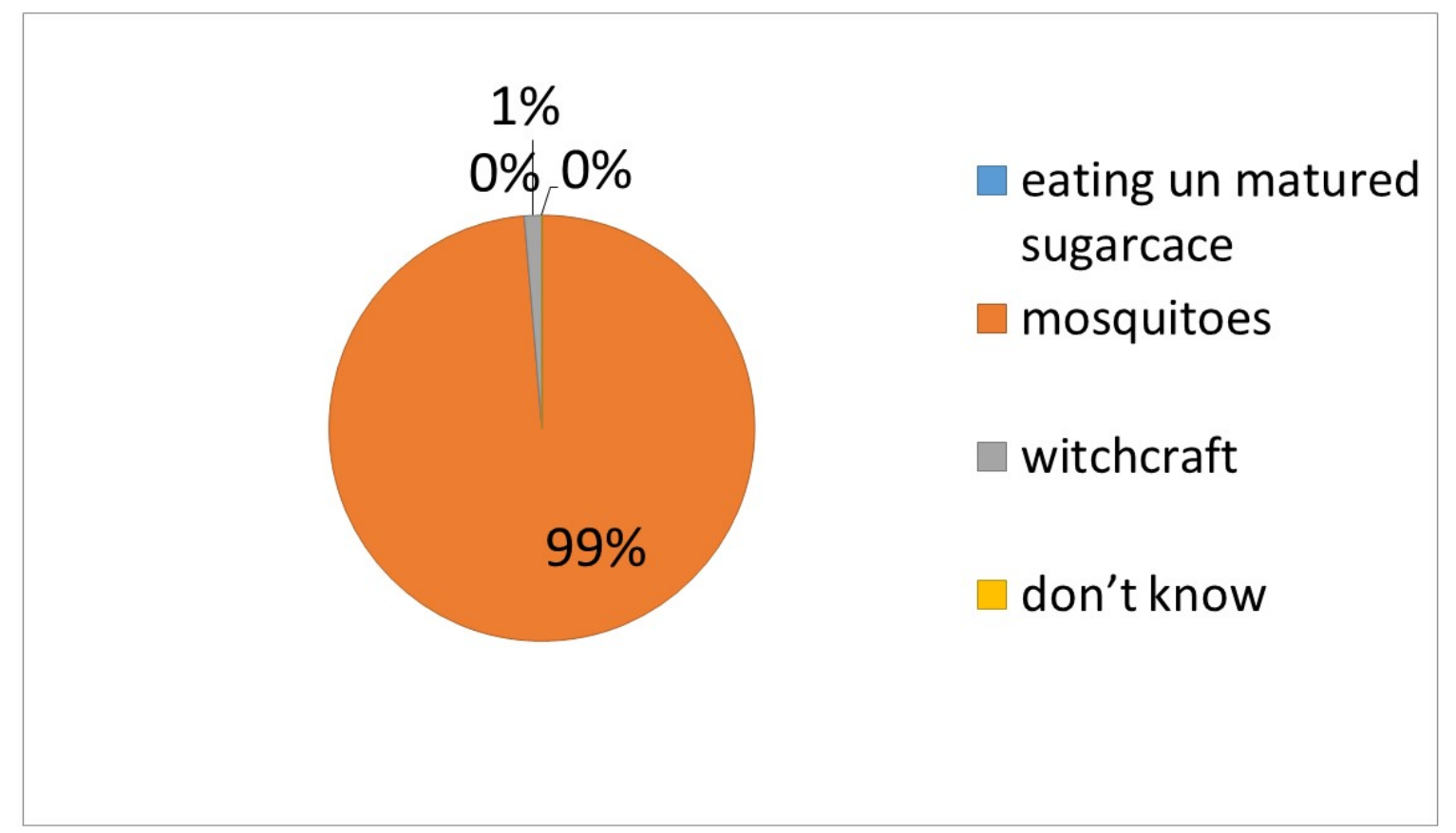

Chart 1. Dangers of Malaria during Pregnancy

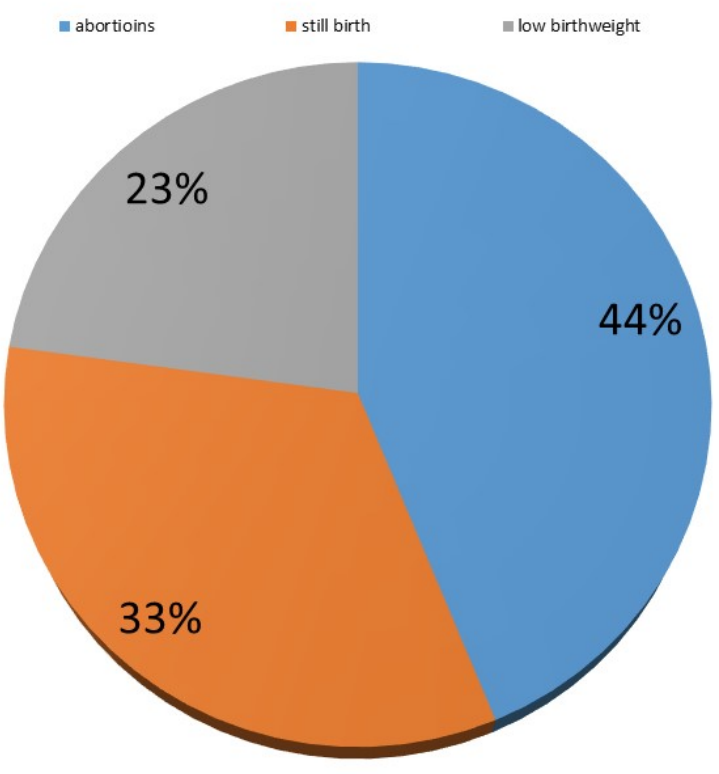

Chart 2. Dangers of Malaria during Pregnancy 
Table 2. distribution of religious affiliations of the study participants

\begin{tabular}{llll}
\hline & & frequency & Percentage \\
RELIGION & Catholic & 25 & 33.3 \\
& Anglican & 20 & 26.7 \\
& Muslim & 17 & 22.7 \\
& Others & 13 & 17.3 \\
\hline
\end{tabular}

Table 3. Distribution of Marital Status and Parity of the Study Participants

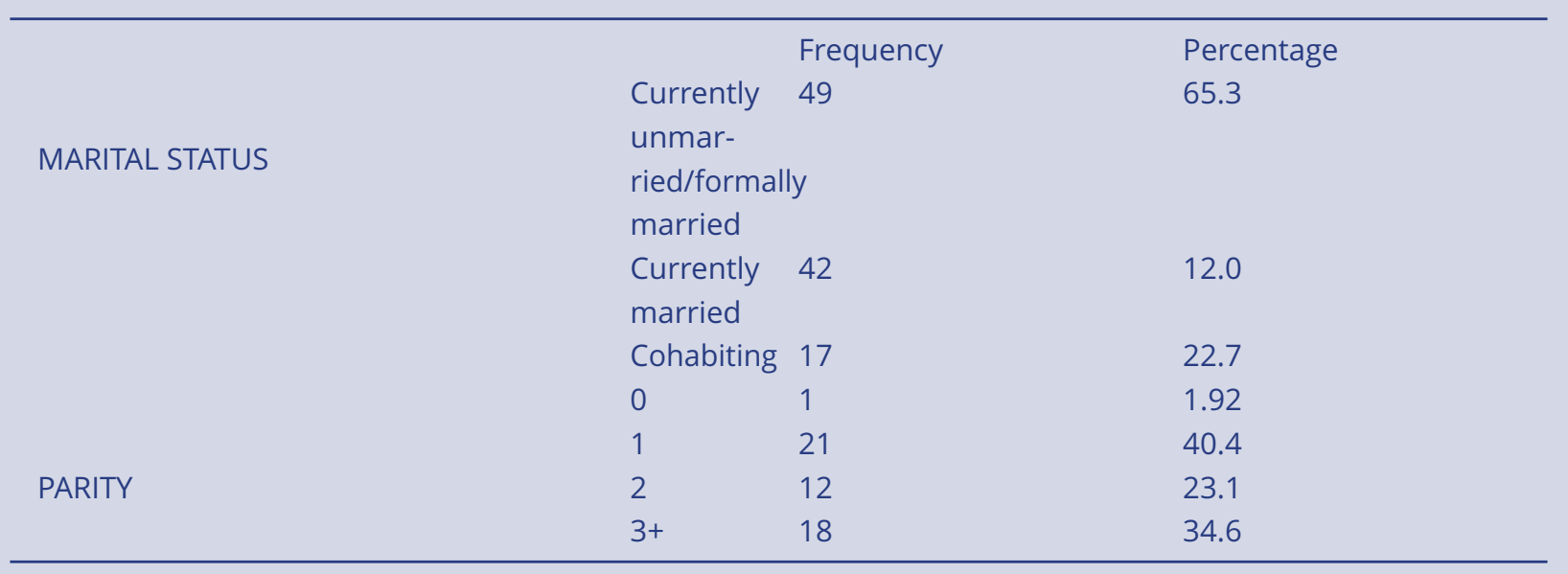

Table 4. Distribution of Religious Affiliations of the Study Participants

\begin{tabular}{|c|c|c|c|}
\hline \multirow{5}{*}{ OCCUPATION } & & frequency & Percentage \\
\hline & Government/non- & 14 & 18.7 \\
\hline & $\begin{array}{l}\text { government employ- } \\
\text { ment }\end{array}$ & & \\
\hline & Self-employed & 19 & 25.3 \\
\hline & Unemployed/student & 42 & 56 \\
\hline
\end{tabular}

11 Figure 4: Whether the Participants Got Any Side Effects after Taking Fasindar /Coartem/Duocotexin

12 Figure 6: Number of Fasindar Doses Taken by Participants during this Pregnancy

13 Figure 7: wheather the drugs always available at the health facility?
14 Figure 8: frequency of sleeping under a mosquito net last month

Individual and Service-Related Factors Associated With IPTp Uptake

Logistic regression (Table 2 ) was used to assess the level of association between the variables that showed a significant effect on the uptake of IPTp. Results from the logistic regression indicated that respondents who had heard about IPTp were 4.89 times more likely to take up IPTp compared to those who had not heard about it (OR 4.89, 95\% Cl: 1.1520.87). Also, respondents who had more than 3 ANC visits increased their uptake of IPTp compared to those with one ANC visit (OR 25, 95\% Cl: 2.2284). The gestational age of the respondents was 


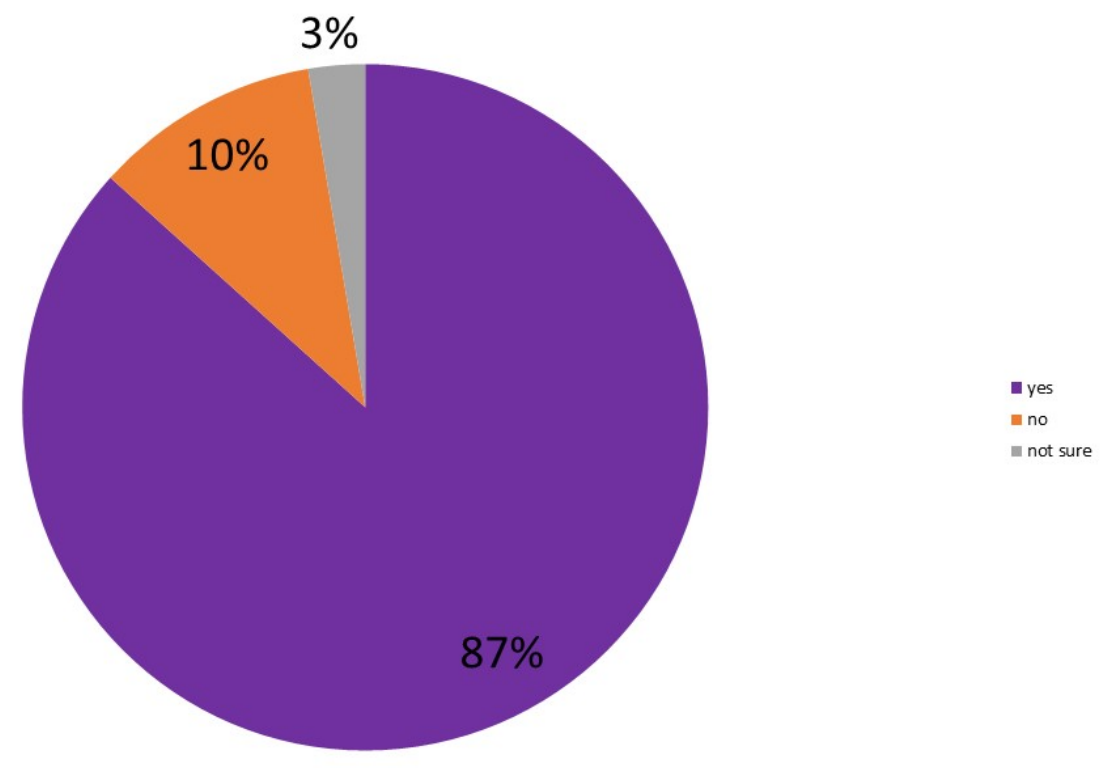

Chart 3. Uptakeof IPTp by Participants during the Current Pregnancy

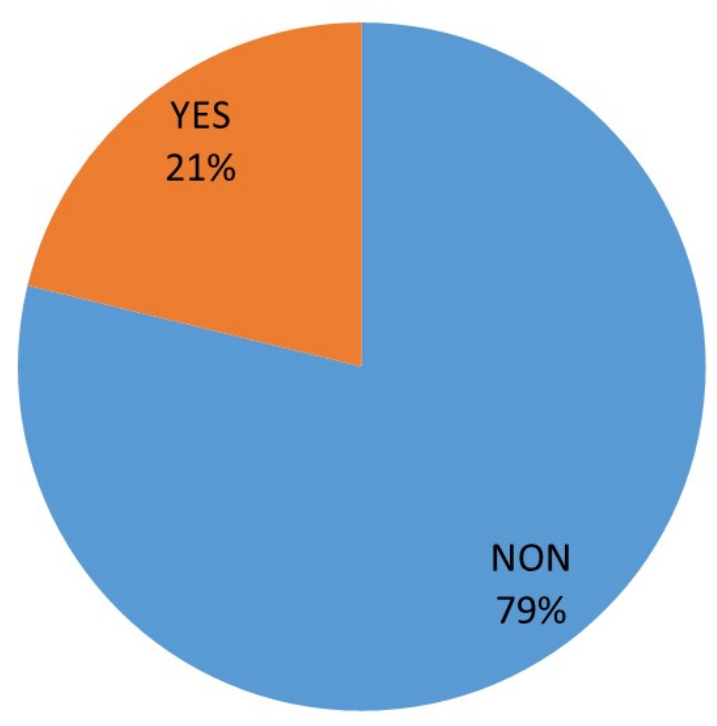

Chart 4. Side Effects of Fasindar / Coartem / Duocoxetecin Reported By Participants 


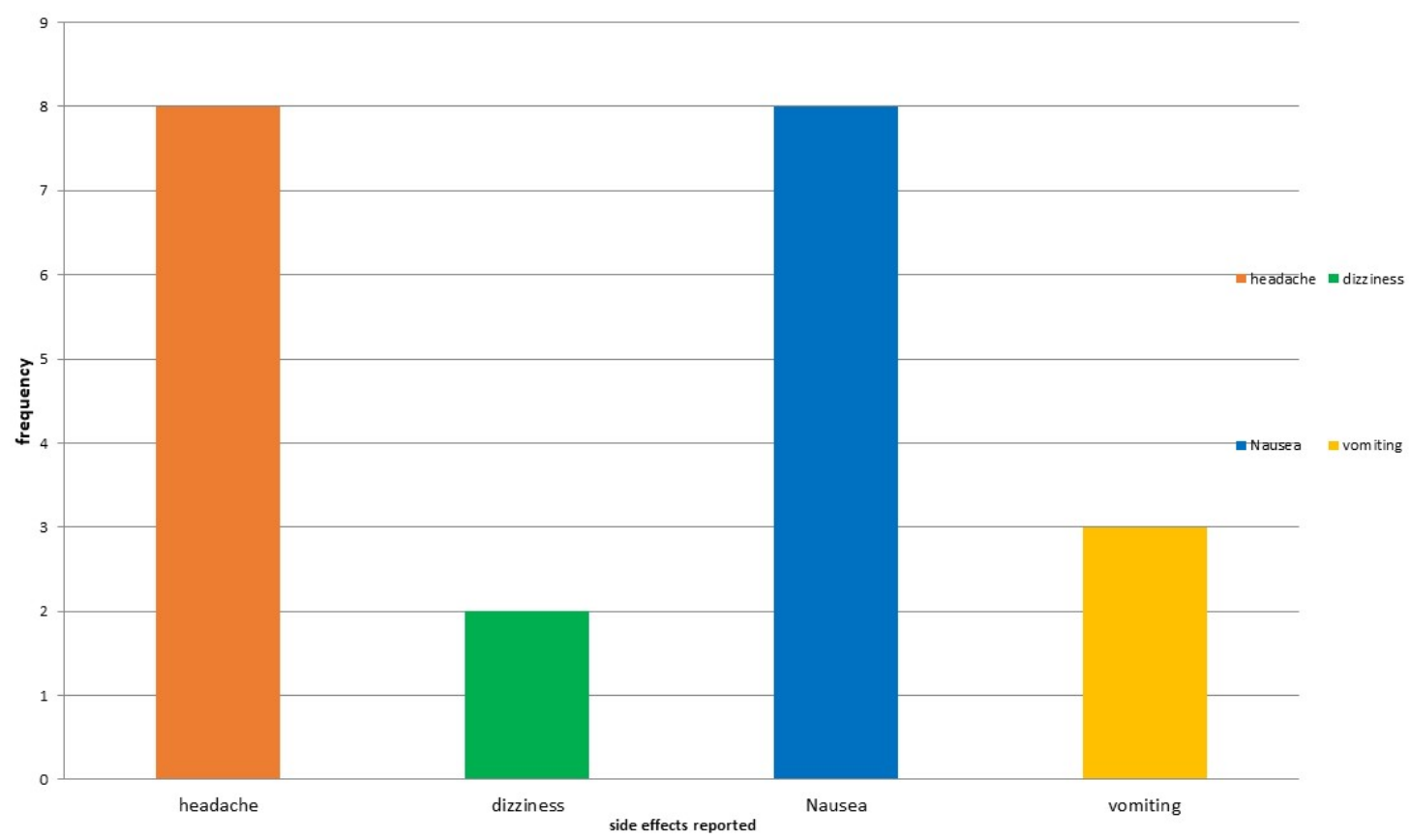

Chart 5. Side Effects of Fasindar / Coartem /Duocoxetecin Reported By Participants

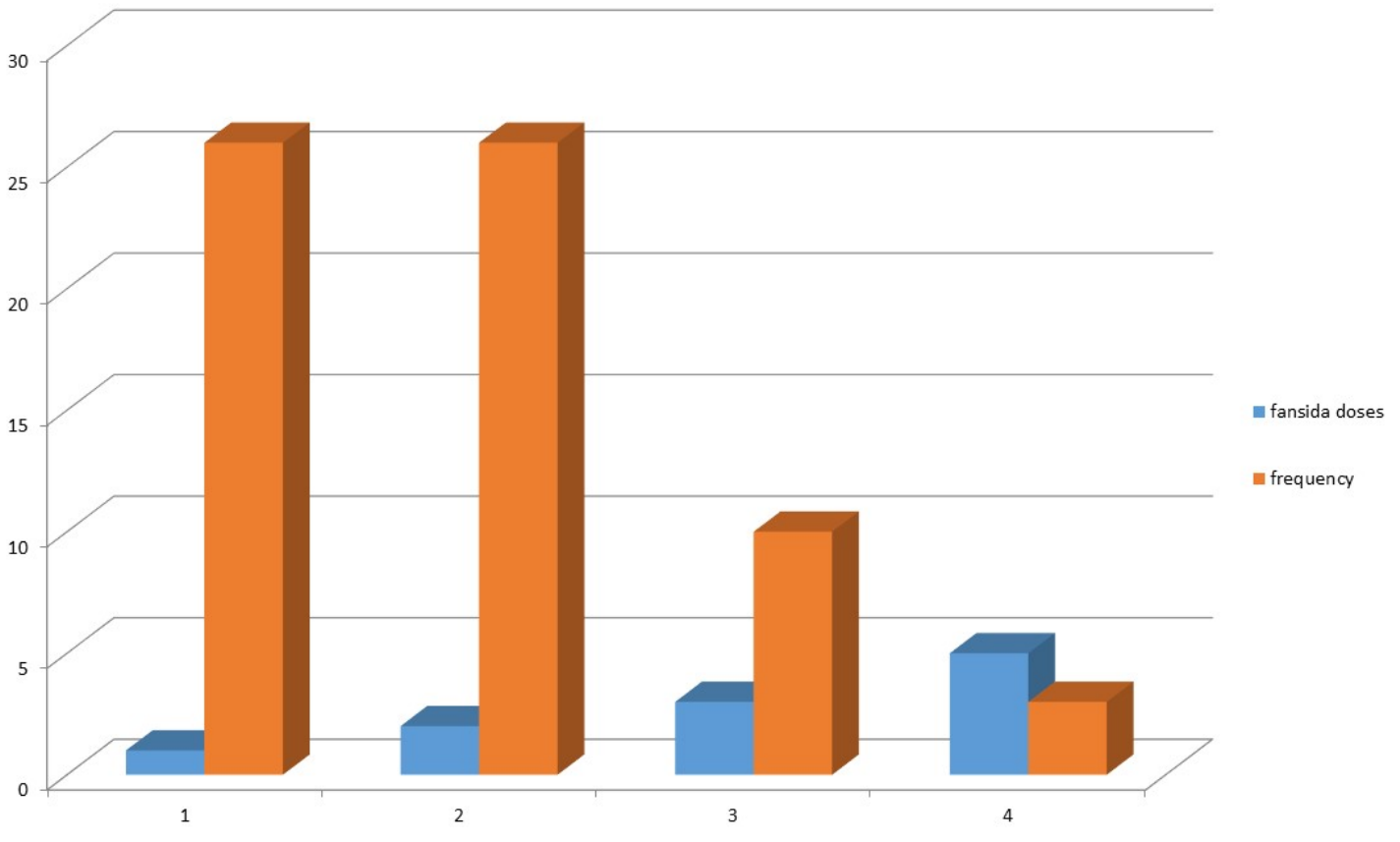

Chart 6. Numberof Fasindar Doses Taken by Participants during this Pregnancy 


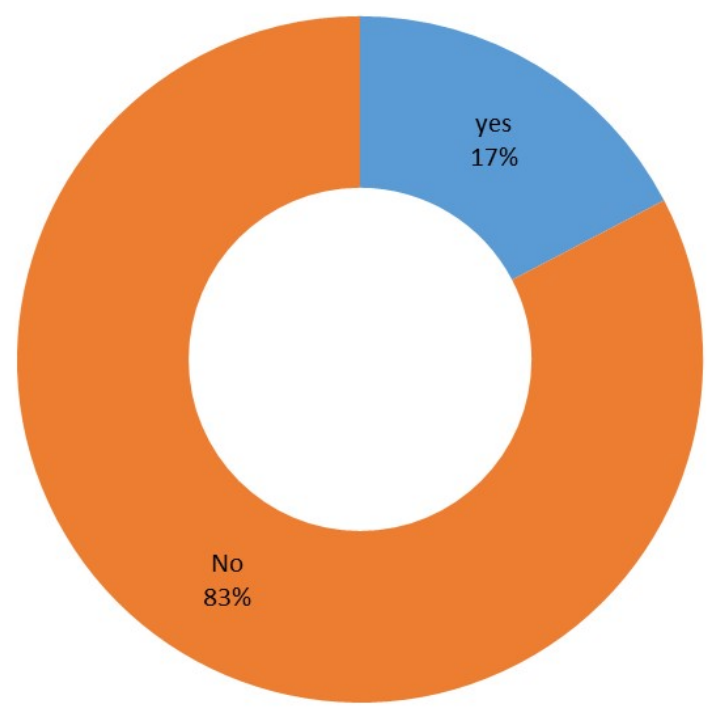

Chart 7. wheather the drugs always available at the health facility?

also strongly associated with the uptake of IPTp. The respondents in the 3rd trimester were 27.3 more likely to take up IPTp compared to those in the 1st trimester. Age, educational level, religion, marital status, occupation, parity, the timing of first ANC visit, and knowledge about pregnancy-related dangers of malaria did not influence the uptake of IPTp in bivariate analysis.

After adjusting for other variables in the model, however, occupation, gestational age, number of ANC visits, and information about IPT remained the significant predictors of IPTp uptake as shown in (table 2). The unemployed/students were 201 times more likely to take up IPTp compared to the government/non-government workers keeping all other factors uniform (OR 201.06 95\%Cl; 1.68 - 24129.67). Participants who were in the 3rd trimester were taking up IPTp compared to those in the 1st trimester keeping all other factors constant (OR 1328.03 95\% Cl2.97 - 593825.4). keeping all other variables constant, participants who were in their 3rd ANC visit were taking up IPTp compared to those who had attended one visit (OR 5631.445 $95 \% \mathrm{Cl} 2.57-1230.00$ )

Research Question Two: What was the prevalence of malaria among Pregnant Women Attending ANC at Kasanje Center III

\section{Incidence of malaria among the study participants}

Of the $75 \%$ participants, 38(50.7\%) who had their blood tested for malaria, all the 38 participants had negative malaria results. Thus, the incidence of malaria in the study participants was zero.

\section{Discussion, Conclusion, and Recommendation \\ 17 Discussion of Results.}

Research Question One: What were the Factors Associated with IPTp Uptake among Pregnant Women Attending ANC at Kasanje Health Center III, Wakiso District?

A cross-sectional survey was carried out to explore the extent of IPTp uptake and associated factors among pregnant women attending ANC at Kasanje Health Center III. The outcome of the survey revealed that the majority of the participants $(79 \%)$ were above 20 years a mean (+/-SD) age of 25.6 (+/- 6.5) years. This demonstrates that most respondents were mature people and would thus get pregnant.

The survey also revealed that $62.67 \%$ of the participants knew about IPTp, ways how malaria is transmitted, and the main complications of malaria in pregnancy. This could be a result that most of 


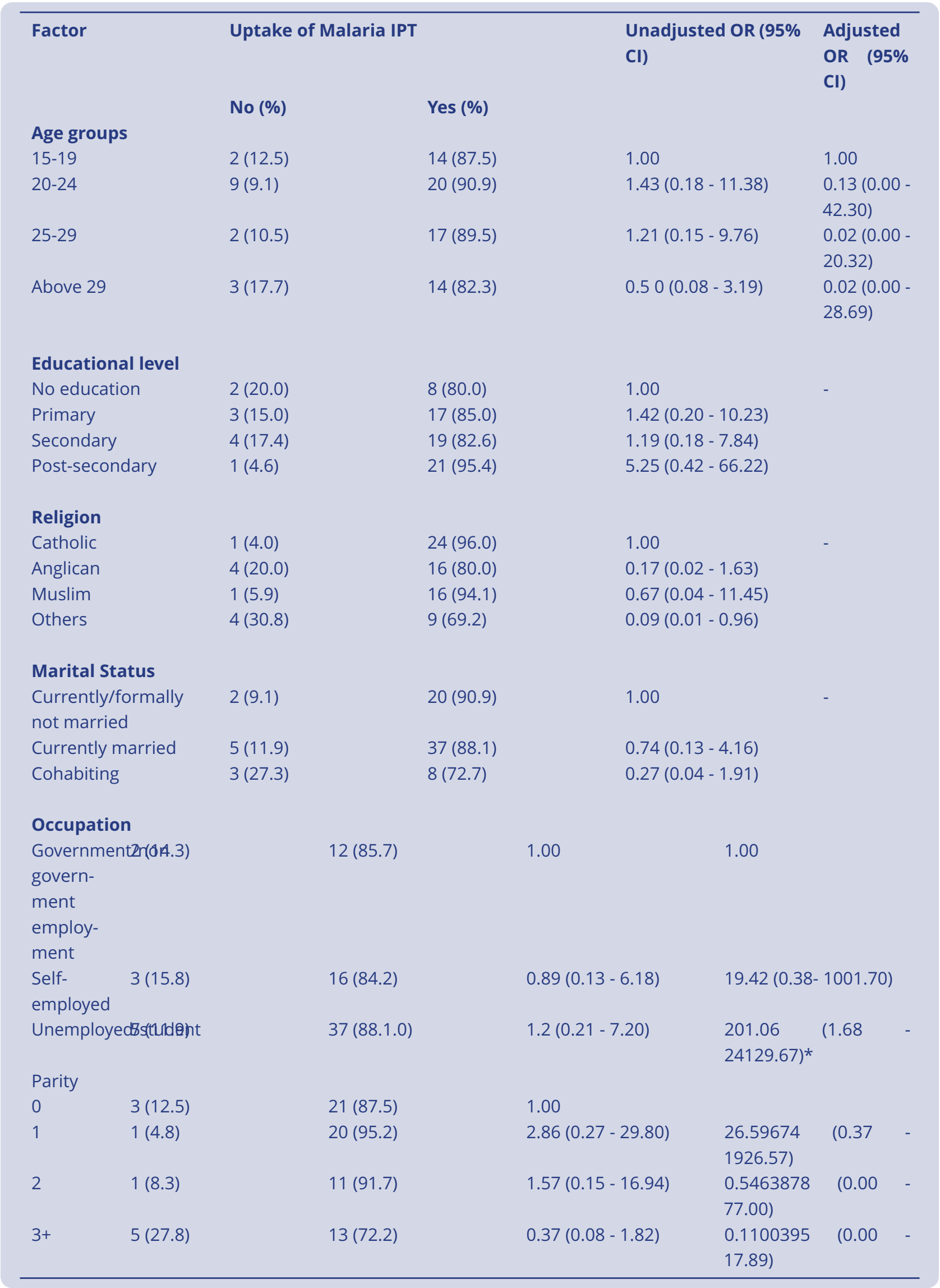




\begin{tabular}{|c|c|c|c|c|}
\hline \multirow[t]{3}{*}{ Factor } & \multicolumn{2}{|c|}{$\begin{array}{l}\text { Uptake of } \\
\text { Malaria IPT }\end{array}$} & \multirow[t]{3}{*}{$\begin{array}{l}\text { Unadjusted OR } \\
(95 \% \mathrm{CI})\end{array}$} & \multirow[t]{3}{*}{$\begin{array}{l}\text { Adjusted OR (95\% } \\
\mathrm{CI})\end{array}$} \\
\hline & No & Yes & & \\
\hline & $(\%)$ & $(\%)$ & & \\
\hline \multicolumn{5}{|l|}{ Pregnancy age } \\
\hline $0-13$ weeks & $\begin{array}{l}2 \\
(66.7)\end{array}$ & $1(33.3)$ & 1.00 & 1.00 \\
\hline 14 - 26 weeks & $\begin{array}{l}5 \\
(17.9)\end{array}$ & $\begin{array}{l}23 \\
(82.1)\end{array}$ & $9.2(.069-122.38)$ & $17.75(0.35$ - 913.58) \\
\hline 27 - 40 weeks & $\begin{array}{l}3 \\
(6.8)\end{array}$ & $\begin{array}{l}41 \\
(93.2)\end{array}$ & $\begin{array}{l}27.33(1.89- \\
395.25)^{\star}\end{array}$ & $\begin{array}{l}1328.03(2.97- \\
593825.4)^{*}\end{array}$ \\
\hline \multicolumn{5}{|l|}{ Timing of first ANC } \\
\hline 1 Months & $\begin{array}{l}5 \\
(15.2)\end{array}$ & $\begin{array}{l}28 \\
(84.8)\end{array}$ & 1.00 & \\
\hline 2 months & $\begin{array}{l}2 \\
(8.7)\end{array}$ & $\begin{array}{l}21 \\
(91.3)\end{array}$ & $1.66(.033-10.63)$ & - \\
\hline 3 months & $\begin{array}{l}0 \\
(0.0)\end{array}$ & $\begin{array}{l}10 \\
(100.0)\end{array}$ & - & \\
\hline 4 months & $\begin{array}{l}2 \\
(40.0)\end{array}$ & $3(60.0)$ & $0.27(0.04-2.03)$ & \\
\hline 5 months & $\begin{array}{l}0 \\
(0.0)\end{array}$ & $\begin{array}{l}1 \\
(100.0)\end{array}$ & - & \\
\hline don't know & $\begin{array}{l}1 \\
(33.3)\end{array}$ & $2(60.7)$ & $0.36(0.03-4.72)$ & \\
\hline \multicolumn{5}{|l|}{ Number of ANC visit } \\
\hline 1 & $\begin{array}{l}4 \\
(50.0)\end{array}$ & $4(50.0)$ & 1.00 & 1.00 \\
\hline 2 & $\begin{array}{l}4 \\
(17.4)\end{array}$ & $\begin{array}{l}19 \\
(82.6)\end{array}$ & $4.75(0.82-27.50)$ & $\begin{array}{l}3.183324(0.07- \\
149.52)\end{array}$ \\
\hline 3 & $\begin{array}{l}1 \\
(5.6)\end{array}$ & $\begin{array}{l}17 \\
(94.4)\end{array}$ & $\begin{array}{l}17.0(1.47-196 \\
.43)^{\star}\end{array}$ & $\begin{array}{l}5631.445(2.57- \\
1230.00)^{*}\end{array}$ \\
\hline More than 3 & $\begin{array}{l}1 \\
(3.9)\end{array}$ & $\begin{array}{l}25 \\
(96.1)\end{array}$ & $25(2.20-284.61)^{\star}$ & $\begin{array}{l}22.90817(0.24- \\
2231.26)\end{array}$ \\
\hline \multicolumn{5}{|l|}{ Information about IPT } \\
\hline No & $\begin{array}{l}7 \\
(25.0)\end{array}$ & $\begin{array}{l}21 \\
(75.0)\end{array}$ & 1.00 & 1.00 \\
\hline Yes & $\begin{array}{l}3 \\
(6.4)\end{array}$ & $\begin{array}{l}44 \\
(93.6)\end{array}$ & $4.89(1.15-20.82)^{*}$ & $\begin{array}{l}26.31159(1.06- \\
651.78)^{\star}\end{array}$ \\
\hline \multicolumn{5}{|c|}{$\begin{array}{l}\text { Knowledge about pregnancy related } \\
\text { dangers of malaria }\end{array}$} \\
\hline Don't know any danger & $\begin{array}{l}0 \\
(0.0)\end{array}$ & $\begin{array}{l}1 \\
(100.0)\end{array}$ & 1.00 & \\
\hline Knows single danger & $\begin{array}{l}2 \\
(9.5)\end{array}$ & $\begin{array}{l}19 \\
(90.5)\end{array}$ & $0.89(0.14-5.82)$ & \\
\hline Knows two dangers & $\begin{array}{l}5 \\
(27.8)\end{array}$ & $\begin{array}{l}13 \\
(72.2)\end{array}$ & $0.24(0.05-1.17)$ & \\
\hline Knows three dangers & $\begin{array}{l}3 \\
(8.6)\end{array}$ & $\begin{array}{l}32 \\
(91.4)\end{array}$ & - & \\
\hline
\end{tabular}




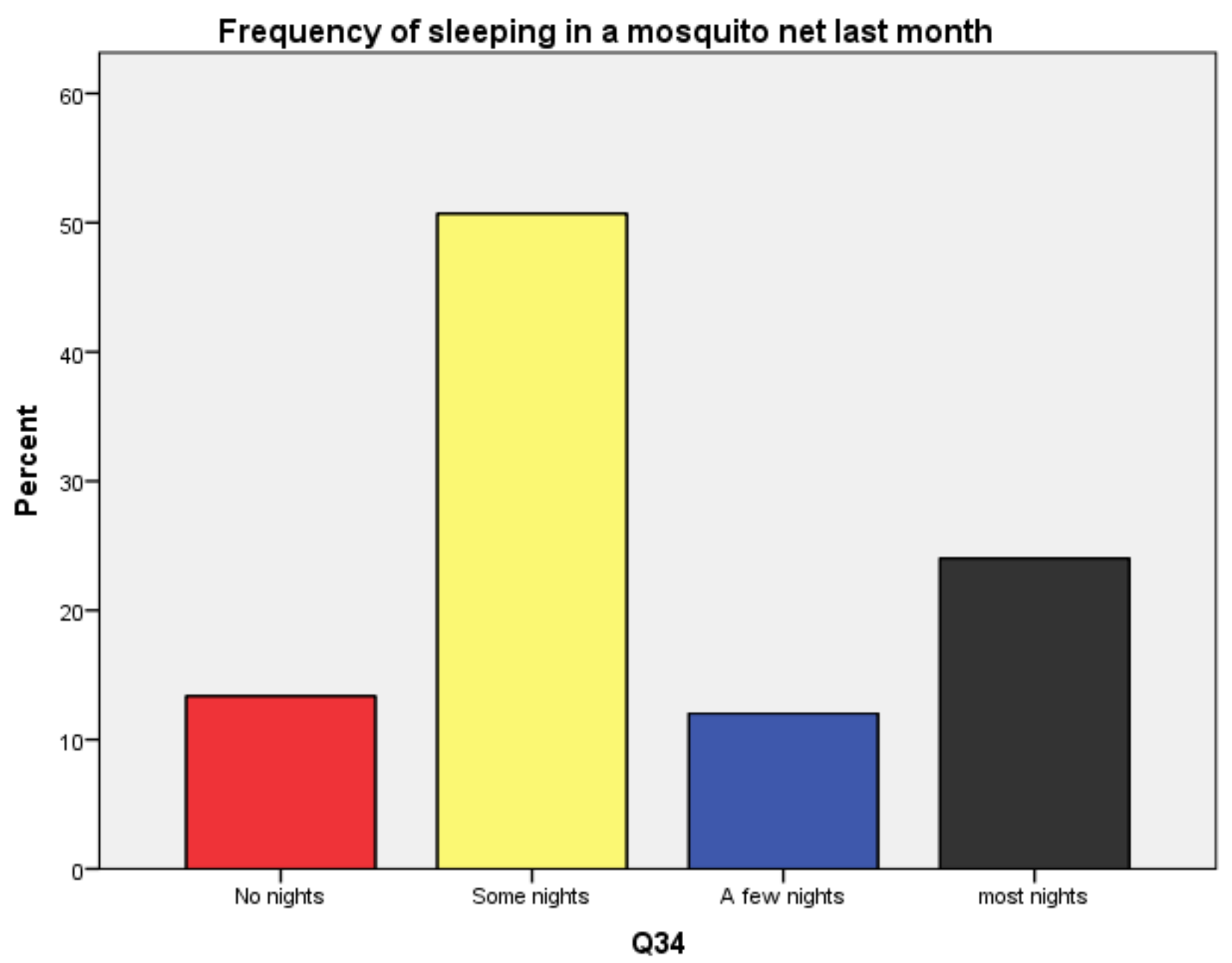

Figure 1. frequencyof sleeping under a mosquito net last month

the participants had attained secondary level education and above and hence they could have attained the knowledge from the basic secondary curriculum. In addition to this, all the participants had attained at least one ANC visit, and yet most were attended to by the trained health professionals (mainly midwives) that are trained to avail such information to the patients. The malaria knowledge about its complications is in line with a study done by Bauserman(Bauserman et al., 2019).

The biggest number of participants (86.67\%) reported being utilizing IPTp during the current pregnancy although some few (21\%) reported getting side effects after taking fasindar/ coartem/ duocotexin. Those who reported having side effects reported headache and nausea as the main side effects. This is also documented in most books of pharmacology as headache and nausea being the main reported side effects of fasindar. This is also in line with a study done by (Hill et al., 2013) who found out that the side effects of medications used in IPTp were a hindrance in the taking up of IPTp. However, $13.33 \%$ of the participants who missed IPTp gave those reasons for drug stock-outs and reporting late for their first antenatal visit.

Most of the participants had taken 1 or 2 doses of fasindar because many of them were in the 2 nd ANC visit and hence could not have received more than 2 doses of fansida. This is in line with the WHO recommendations that all pregnant women should receive at least 3 doses of fansida starting as early as possible in the second trimester(WHO, 2013).

After adjusting for other variables in the logistic model, occupation, gestational age, several ANC visits, and information about IPTp remained the significant predictors of IPTp uptake. This is in line with the study done by (Muhumuza, Namuhani, Balugaba, Namata, \& Kiracho, 2016) 
Research Question Two: What was the Prevalence of Malaria among Pregnant Women Receiving IPTp in ANC at Kasanje Health Care III, Wakiso District?

Out of the $75 \%$ participants, $38(50.7 \%)$ who had their blood tested for malaria, all had negative malaria results. Thus, the incidence of malaria in the study participants was zero. Despite the knowledge about malaria transmission and its complications in pregnancy, most of the participants(50\%) were sleeping in the mosquito nets for some nights in the previous month (figure 8 ) and most of them had received 1 or 2 doses of IPTp by the time of testing.

\section{Conclusion}

The current study that was exploring malaria intermittent preventive treatment (IPTp) uptake among pregnant women attending antenatal care at Kasanje Health Center III, established that increased number of ANC visits, providing IPTp information to pregnant women and the gestational age of pregnancy resulted in increased uptake of IPTp and thus reduced incidences of malaria cases.

\section{Recommendations}

The ministry of health should continue to maintain the IPTp programs in all health facilities with antenatal services.

All pregnant mothers should be tested for malaria on every antenatal visit and long-lasting insecticide-treated mosquito nets should be provided.

Health education talks to mothers during antenatal should aim at providing information on IPTp use.

\section{Implications for Nursing Practice}

The implications of these findings to the nursing practice include the following: Nurses and midwives have a responsibility of providing all the necessary information about IPTp to mothers and caretakers to reduce malaria incidences in pregnant mothers. They also need to encourage mothers on IPTp use.

\section{Acknowledgment}

I would like to acknowledge my family for having supported me in this course because all I needed was psychosocial support which I got from them. I acknowledge my supervisor Stewart Ssentayi for the support rendered to me and the entire research.

\section{List of Abbreviations}

IPTp- Intermittent prevention treatment prophylaxis

ANC-Antenatal Care

WHO-World Health Organization

ITNs- Insecticide Treated Nets

DOT-Direct Observed Therapy

SP-Sulfadoxine/Pyrimethamine

DHA-PQ- Dihydroartemisinin-piperaquine

UBOS- Uganda Bureau of Statistics

ICF-Informed Consent Form

UDHS-Uganda Demographic Health Survey

FANC-Focused Antenatal Care

PNFP-Private Non For Profit

SBCC-Social Behavioral Change Communication

USAID- United States Agency for International

Development

MIP- Malaria in Pregnancy

VHTs- Village Health Teams

ACTs- Artemisinin-based Combination Treatments

INTs- Insecticide Treated Nets

\section{A References:}

1) African Strategies for Health. (2016). Facility Level Factors Influencing The Uptake Of Intermittent Preventative Therapy For Malaria In Pregnant Women. Report on a formative assessment conductedin Uganda Retrieved from 4301 N. Fairfax Drive, Suite 400, Arlington, VA 22203 https://www.msh.org/sites/msh.org/files/ip tp_assessment_sep2016_final_0.pdf

2) Agarwal, K., Alonso, P., Chico, R. M., Coleman, J., Dellicour, S., Hill, J., . . M Mitchell, K. (2015). Global Call to Action to scale-up coverage of intermittent preventive treatment of malaria in pregnancy: seminar report: BioMed Central. https://doi.org/10.118 6/s12936-015-0730-3

3). Bauserman, M., Conroy, A. L., North, K., Patterson, J., Bose, C., \& Meshnick, S. (2019). An overview of malaria in pregnancy. Paper presented at the Seminars in perinatology https://doi.org/10.1053/j. semperi.2019.03.018

4) Brentlinger, P. E., Dgedge, M., Correia, M. A. C., Rojas, A. J. B., Saúte, F., Gimbel-Sherr, K. H., . . . Gloyd, S. (2007). Intermittent preventive treatment of malaria during pregnancy in central 
Mozambique. Bulletin of the World Health Organization, 85(11), 873-879. doi:10.2471/blt.06.033381 https://doi.org/10.2471/BLT.06.033381

5) Ekejindu, I., Udigwe, G., \& Chijioke, I. (2006). Malaria and anaemia in pregnancy in Enugu, south east Nigeria. African journal of medicine and medical sciences, 35(1), 1-3

6) Guinovart, C., Navia, M., Tanner, M., \& Alonso, P. (2006). Malaria: burden of disease. Current molecular medicine, 6(2), 137-140 https://doi.org/10.217 4/156652406776055131

7) Hill, J., Hoyt, J., van Eijk, A. M., D'Mello-Guyett, L., ter Kuile, F. O., Steketee, R., . . Webster, J. (2013). Factors affecting the delivery, access, and use of interventions to prevent malaria in pregnancy in sub-Saharan Africa: a systematic review and metaanalysis. PLoS Medicine, 10(7), e1001488 https://d oi.org/10.1371/journal.pmed.1001488

8) Lagerberg, R. E. (2008). Malaria in pregnancy: a literature review. Journal of midwifery \& women's health, 53(3), 209-215 https://doi.org/10.1016/j.jm wh.2008.02.012

9) Mubyazi, G. M., Magnussen, P., Goodman, C., Bygbjerg, I. C., Kitua, A. Y., Olsen, O. E., Byskov, J., Hansen, K. S., \& Bloch, P. (2008). Implementing Intermittent Preventive Treatment for Malaria in Pregnancy: Review of Prospects, Achievements, Challenges and Agenda for Research. The open tropical medicine journal, 1, 92-100. https://doi.org /10.2174/1874315300801010092

10) Muhumuza, E., Namuhani, N., Balugaba, B. E., Namata, J., \& Kiracho, E. E. (2016). Factors associated with use of malaria control interventions by pregnant women in Buwunga subcounty, Bugiri District. Malaria journal, 15(1), 342 https://doi.org/ 10.1186/s12936-016-1407-2

11) Ndyomugyenyi, R., Neema, S., \& Magnussen, P. (1998). The use of formal and informal services for antenatal care and malaria treatment in rural Uganda. Health policy and planning, 13(1), 94-102 https://doi.org/10.1093/heapol/13.1.94

12) Ouma, P. O., Van Eijk, A. M., Hamel, M. J., Sikuku, E. F., Odhiambo, K., Munguti, J. G., Slutsker. (2007). The effect of health care worker training on the use of intermittent preventive treatment for malaria in pregnancy in rural western Kenya. Tropical Medicine \& International Health, 12(8), 953961 https://doi.org/10.1111/j.1365-3156.2007.018 76.x

13). Poespoprodjo, J. R., Fobia, W., Kenangalem, E., Lampah, D. A., Sugiarto, P., Tjitra, E., . . Price,
R. N. (2014). Dihydroartemisinin-piperaquine treatment of multidrug resistant falciparum and vivax malaria in pregnancy. PloS one, 9(1), e84976 https: //doi.org/10.1371/journal.pone.0084976

14) Ricci, F. (2012). Social implications of malaria and their relationships with poverty. Mediterranean journal of hematology and infectious diseases, 4(1). https://doi.org/10.4084/mjhid.2012.04 8

15) Sangaré, L. R., Stergachis, A., Brentlinger, P. E., Richardson, B. A., Staedke, S. G., Kiwuwa, M. S., \& Weiss, N. S. (2010). Determinants of use of intermittent preventive treatment of malaria in pregnancy: Jinja, Uganda. PLoS One, 5(11), e15066-e15066. doi:10.1371/journal.pone.0015066 https://doi.org /10.1371/journal.pone.0015066

16) Schantz-Dunn, J., \& Nour, N. M. (2009). Malaria and pregnancy: a global health perspective. Reviews in obstetrics \& gynecology, 2(3), 186-192.

17) Uganda Bureau of Statistics (UBOS) \& ICF. (2018). Uganda Demographic and Health Survey 2016 Kampala Uganda and Rockville, Maryland, USA: UBOS and ICF

18) USAID. (2019). President's Malaria Initiative Uganda, Malaria Operational Plan FY 2019. Retrieved from https://www.pmi.gov/docs/default-so urce/default-document-library/malaria-operation al-plans/fy19/fy-2019-uganda-malaria-operational -plan.pdf?sfvrsn=3

19) WHO. (2013). WHO policy brief for the implementation of intermittent preventive treatment of malaria in pregnancy using sulfadoxinepyrimethamine (IPTp-SP). Retrieved from https://w ww.who.int/malaria/publications/atoz/iptp-sp-up dated-policy-brief-24jan2014.pdf?ua=1

20) WHO. (2013). WHO policy brief for the implementation of intermittent preventive treatment of malaria in pregnancy using sulfadoxinepyrimethamine (IPTp-SP). Retrieved from https://w ww.who.int/malaria/publications/atoz/iptp-sp-up dated-policy-brief-24jan2014.pdf?ua=1

21) WHO. (2018a). Implementing malaria in pregnancy programs in the context of World Health Organization recommendations on antenatal care for a positive pregnancy experience. Retrieved from https://www.who.int/reproductivehealth/pu blications/implementing-malaria-pregnancy-prog rammes-brief/en/

22) York A. (2015). Reducing the risk of malaria in pregnancy in Uganda - observations from the field. https://www.malariaconsortium.org/blog/re 
ducing-the-risk-of-malaria-in-pregnancy-in-ugand a-observations-from-the-field/ 